\title{
A New Method for Current Differential ISFET/REFET Readout Circuit
}

\author{
Taksin Nobpakoon, Wanchai Pijitrojana, and Amporn Poyai
}

\begin{abstract}
This paper presents a new differential current mode readout circuit for ion sensitive field effect transistor (ISFET) to be used as a $\mathrm{pH}$ sensor. The circuit used only two MOSFETs to provide current differential pairs result in simplification and very small circuit. It provides a linear sensitivity to $\mathrm{pH}$ over a wide range (pH 2-12) which is 25.6 $\mu \mathrm{A} / \mathrm{pH}$ with power dissipation of $937.6 \mu \mathrm{W}$ at $270 \mathrm{C}$. The circuit is operating in differential mode, reference field effect transistor (REFET) is provided to compensate environment conditions. Temperature compensation in a range of $22-320 \mathrm{C}$ is investigated. It can be well compensated output varying. Simulation results validate the performance of the circuit.
\end{abstract}

Index Terms-ISFET readout circuit, MOSFET differential pairs, current mode, small circuit

\section{INTRODUCTION}

The ion sensitive field effect transistor (ISFET) is a chemically sensitive field effect transistor was introduced by Bergveld in the 1970s. The ISFET is similar to a MOSFET, except that the gate of MOSFET is replaced by an ion sensitive membrane exposed to an aqueous solution [1]. Fig. 1 presents schematic diagram of ISFET and MOSFET. The membrane is sensitive to hydrogen ions in the test solution when there is a change in hydrogen ions on the membrane surface, a change in the potential on the surface will occur and it is correlated to solution $\mathrm{pH}$, causes a modulation in the ISFETs threshold voltage. The ISFET sensors have many advantages, particularly attractive from their small size, robustness, low power consumption, capability to integrate sensor and signal processing unit in single chip, simplicity in batch fabrication and low cost.

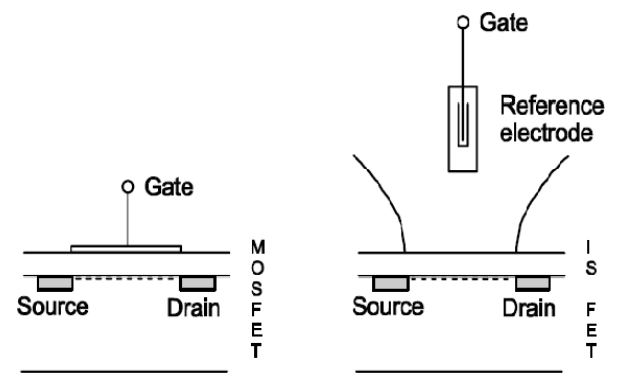

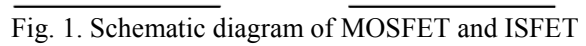

Manuscript received August 15, 2012; revised October 12, 2012.

Taksin Nobpakoon and Wanchai Pijitrojana are with the Electrical and Computer Engineering Department Faculty of Engineering, Thammasat University 99 Moo 18, Klongneung, Klonglaung, Pathumthani, Thailand, 12120

Amporn Poyai is with Thai Microelectronics Center (TMEC) National Electronics and Computer Technology Center 51/4 Moo 1, Wangtakien, Muang, Chachoengsao, Thailand, 24000 (e-mail: pimpud@hotmail.com).
Many other research works [2]-[7] have contributed to the design of readout circuits in order to obtain the measuring signal. One of the main objectives of designing is to compensate the environment conditions by operating in differential mode with REFET.

In this paper, a new ISFET/REFET differential current mode readout circuit has been proposed. This new circuit is developed current differential technique, result in provides simplification and small circuit. ISFET and REFET are connected as same as MOSFET differential pairs, but no tail current source. Drain voltage of them is changed when there is a change in solution $\mathrm{pH}$ or hydrogen ion concentration. The REFET drain voltage biased to its MOSFET pair, but the ISFET drain voltage series with a floating voltage equals drain-source voltage of the MOSFET pair of the REFET, and biased to its MOSFET pair. The difference current of two MOSFETs can be monitored the measurement system that compensated environment conditions. The paper is organized as follows: Section II presents the operation mechanism and characteristic of ISFET sensor. In section III, the proposed readout circuit is described in detail. In section IV, the simulation results are presented and the conclusion is drawn in section $\mathrm{V}$.

\section{ISFET SENSOR}

The ISFET has the similar operation as that of a conventional MOSFET except that an electrolyte gate is used instead of a metal gate. An electric potential difference will occur at the interface between the gate insulator and the solution which depends on hydrogen ion concentration of the solution or $\mathrm{pH}$ value. The change in this potential caused by the $\mathrm{pH}$ changing result in modulated the drain current. The threshold voltage, Vth, of ISFET differs from MOSFET. In ISFET, there is the electrolyte between the gate insulator and the metal gate that is defined as the reference electrode, the threshold voltage is given by [8]:

$$
\begin{gathered}
V_{t h(I S F E T)}=E_{R e f}+\Delta \varphi_{l j}-\varphi_{e o}+\chi_{e o}+ \\
\left(\frac{-\varphi_{s}}{q}-\frac{Q_{o x}+Q_{S S}}{C_{o x}}+2 \varphi_{f}\right)
\end{gathered}
$$

where $E_{R e f}$ is the reference electrode potential, $\varphi_{l j}$ is the liquid-junction potential difference between reference solution and the electrolyte, $\varphi_{e o}$ is the potential of electrolyte-insulator interface, $\chi_{e o}$ is the electrolyte-insulator surface dipole potential, $\varphi_{s} / q$ is the semiconductor work function, $Q_{o x}$ is the semiconductor surface depletion region charge per unit area, $Q_{s s}$ is the fixed surface-state charge density per unit area at the insulator-semiconductor interface, $\mathrm{C}_{\mathrm{ox}}$ is the gate insulator capacitance per unit area, and $\varphi_{\mathrm{f}}$ is the Fermi potential of the semiconductor. Here, the only difference of the terms in the parentheses between ISFET and 
MOSFET is that of absence of the gate metal work function. The other terms in above equation are a group of chemical potential, among which $\varphi_{e o}$ is the only chemical input parameter shown to be a function of the solution $\mathrm{pH}$ value. This chemical dependent characteristic of $\varphi_{e o}$ can be explained by the Hal and Eijkel's theory which is elaborated using the general accepted site-binding model and the Gouy-Chapman-Stern model [8]:

$$
\frac{\partial \varphi_{\mathrm{eo}}}{\partial \mathrm{pH}}=-2.303 \frac{\mathrm{kT}}{\mathrm{q}} \alpha
$$

With $\alpha$ is dimensionless sensitivity parameter, with the value varying between 0 and 1 .

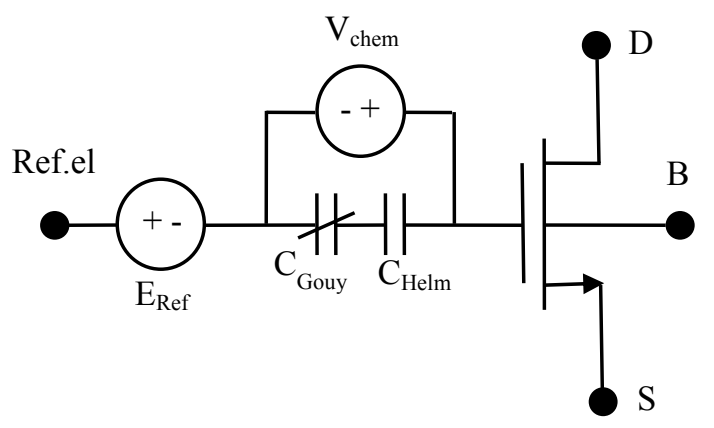

Fig. 2. The ISFET equivalent model

Fig. 2 presents a behavioral macromodel of an n-channel ISFET sensor. While Ref.el, $\mathrm{E}_{\mathrm{Ref}}, \mathrm{D}, \mathrm{S}$, and B stand for the reference electrode, the reference electrode potential, the drain, the source, and the bulk connections, respectively. A voltage source, $V_{\text {chem }}$, is defined as a group of chemical potentials consisting of both $\mathrm{pH}$-dependent potentials, $\varphi_{\mathrm{eo}}$, and $\mathrm{pH}$-independent potentials, $\mathrm{K}$, which consists of $E_{R e f}, \Delta \varphi_{l j}$, and $\chi_{e o}$ in (1). It is given by [8]:

$$
V_{\text {chem }}=-2.303 \frac{k T}{q} \alpha(p H)+K
$$

Assume the n-channel ISFET which $\mathrm{W}=804 \mu \mathrm{m}$ and $\quad \mathrm{L}=$ $18 \mu \mathrm{m}$ is biased as shown in Fig. 3, with $V_{d d}=1 \mathrm{~V}, \quad V_{R e f}=$ $200 \mathrm{mV}, \mathrm{R}_{\mathrm{d}}=300 \Omega$, and the bulk is connected at the source voltage that is grounded. Therefore, drain current, $\mathrm{i}_{\mathrm{d}}$, will be varied follow by change in $\mathrm{pH}$ value. The equations (4) and (5) described the drain voltage, $V_{d}$, and the drain current, $\mathrm{i}_{\mathrm{d}}$, of the ISFET, respectively.

$$
\begin{gathered}
V_{d}=V_{d d}-i_{d} R_{d} \\
i_{d}=\frac{1}{2} K^{\prime}{ }_{n} \frac{W}{L}\left(V_{R e f}-V_{t h}\right)
\end{gathered}
$$

where $\mathrm{W}$ is the width of the channel, $\mathrm{L}$ is the length of the channel, $V_{t h}$ is the threshold voltage, and $\mathrm{K}_{\mathrm{n}}^{\prime}$ is the process trans-conductance parameter.

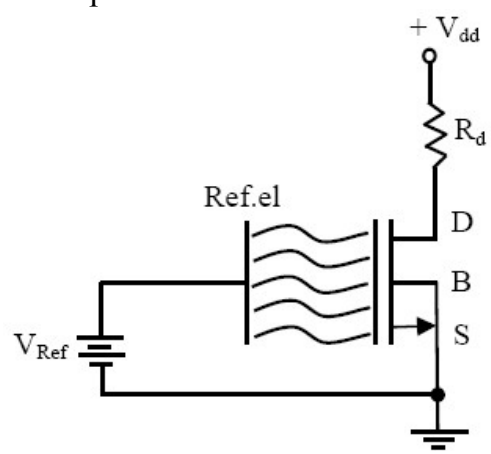

Fig. 3. Schematic diagram of the proposed ISFET operation
Because of the voltage on the reference electrode, $V_{\text {Ref }}$, is kept constant, the change in the solution $\mathrm{pH}$ will led to the change in $\mathrm{I}_{\mathrm{d}}$, and $V_{d}$. The $V_{d}$ versus $\mathrm{pH}$ characterization of the Fig. 3 shown in Fig. 4 which is simulated using the behavioral macromodel of ISFET by HSPICE simulation. The drain voltage will shift right from $823 \mathrm{mV}$ to $948 \mathrm{mV}$ as the $\mathrm{pH}$ value varies from 2 to 12 . The $\mathrm{pH}$ sensitivity is about $13 \mathrm{mV} / \mathrm{pH}$.

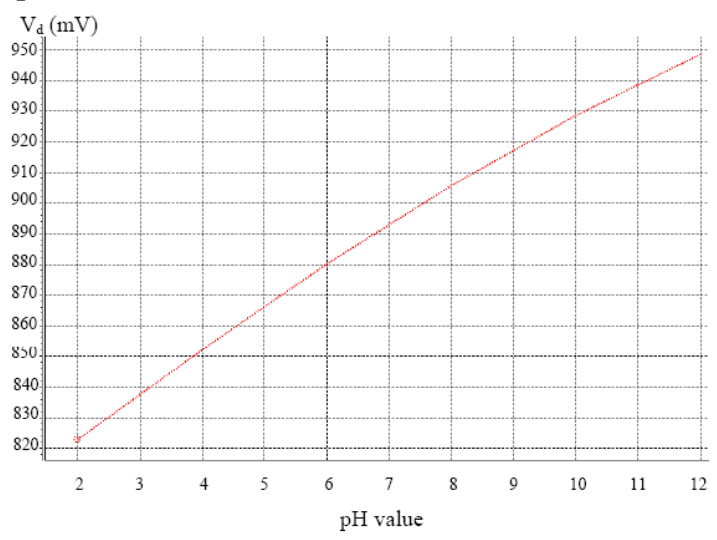

Fig. 4. Characterization of the ISFET operation in fig. 3

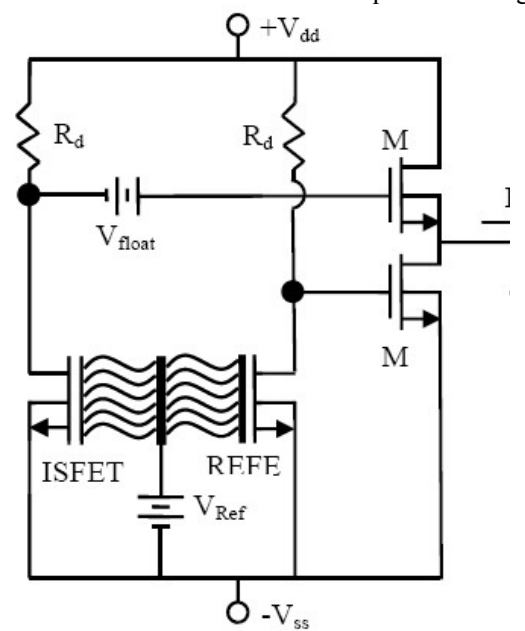

Fig. 5. The proposed differential current mode readout circuit

\section{Proposed Readout Circuit}

The schematic of the proposed ISFET readout circuit is shown in Fig. 5. The basic principle of differential current mode readout circuit is based on Kirchhoff's Current Law that is the total current flowing into a node must be the same as the total current flowing out of the node. The circuit is consists of ISFET, REFET (same design as the ISFET), two matched n-channel MOSFETs, two resistors, $V_{R e f}$, reference electrode, $V_{\text {float }}=$ drain-source voltage of M2, and positive and negative supply. The ISFET operated in a non-saturation region is controlled by constant $V_{\text {Ref. }}$. The two MOSFETs operated in saturation region that is $0<\left(V_{G S}-V_{t h}\right)<V_{D S}$, the drain current can be expressed as follow [5]:

$$
i_{d}=\mu_{n} C_{o x} \frac{W}{2 L}\left(V_{G S}-V_{t h}\right)^{2}
$$

If the ISFET drain current is as same as the REFET, gate-source voltage of two MOSFETs (M1 and M2) will balance. As a result, the drain current of M1 is equal to the drain current of M2; therefore, the ground current is not flowed. If the ISFET drain current is less than the REFET drain current, the gate-source voltage of M1 will more than 
the gate-source voltage of M2. Consequently, the drain current of M1 is more than the drain current of M2, thus the current that cannot flow through M2 will flow to ground. If the ISFET drain current is more than the REFET drain current, the gate-source voltage of M1 will less than the gate-source voltage of M2. As a result, the drain current of M1 is less than the drain current of M2, so the current from ground can flow through M2.

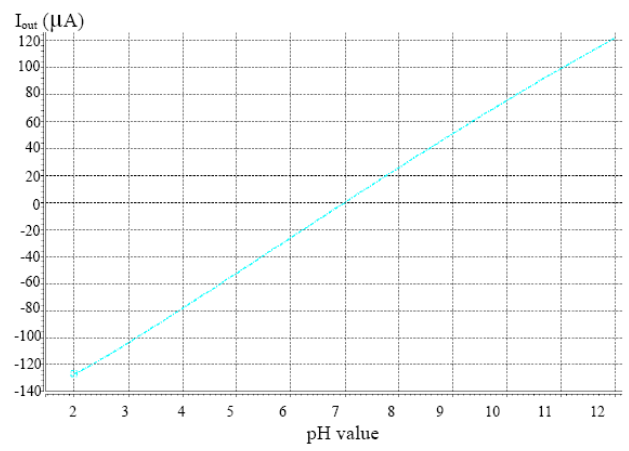

Fig. 6. Output current of the proposed readout circuit

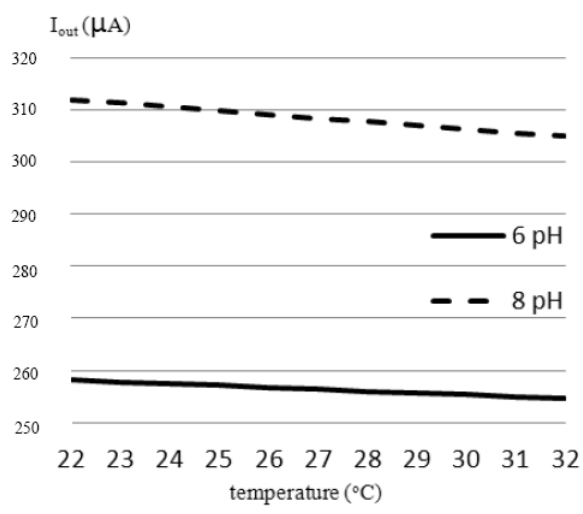

(a) before compensation; output from the ISFET

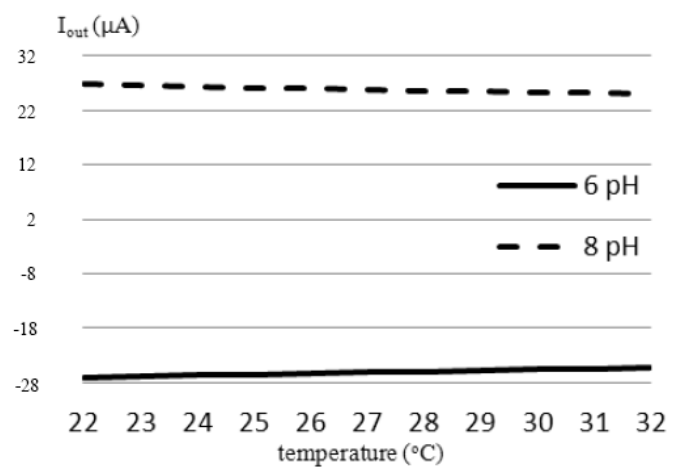

(b) after compensation; output from differential ISFET/REFET Fig. 7. Temperature compensation of the circuit

\section{Simulation Results}

The proposed circuit bas been simulated by HSPICE. The ISFET and REFET were modeled with the behavioral macromodel described in [8], and the BSIM3V3 model parameters of $0.35 \mu \mathrm{m}$ CMOS technology is used to describe the two MOSFETs (M1 and M2). The two MOSFETs have the aspect ratio of $30 \mu \mathrm{m} / 0.5 \mu \mathrm{m}$ whereas the ISFET and REFET have the aspect ratio of $804 \mu \mathrm{m} / 18 \mu \mathrm{m}$. REFET is fixed at $7 \mathrm{pH}$ to show differential operation of the circuit. The proposed circuit parameters are $\quad V d d=+0.5 \mathrm{~V}, V s s=$ $-0.5 \mathrm{~V}$, VRef $=200 \mathrm{mV}$, Vfloat $=0.5 \mathrm{~V}, \quad \mathrm{Rd}=300 \Omega$, and temperature setting is $27 \mathrm{oC}$.

Fig. 6 shows the output current (Iout) of the proposed differential current mode readout circuit when varied the $\mathrm{pH}$ value from 2 to 12 . The output current gives good linearity, the sensitivity to $\mathrm{pH}$ is $25.6 \mu \mathrm{A} / \mathrm{pH}$. This work also do temperature sweep from $22^{\circ} \mathrm{C}$ to $32^{\circ} \mathrm{C}$ under 6 and $8 \mathrm{pH}$, the temperature compensate results are presented in Fig.7. The results claimed that the proposed circuit disturbed by temperature can be well compensated.

\section{CONCLUSIONS}

A new differential current mode readout circuit for ISFET sensor has been presented which promise simplification and small circuit. The circuit structure is simple, it used only two MOSFETs that it can be easy constructed in the very small chip and provided to use in the implantable applications. REFET is used to reduce the temperature effect and environment conditions. The circuit is able to provide a current output linear sensitivity to $\mathrm{pH}$ of $25.6 \mu \mathrm{A} / \mathrm{pH}$ in the range of $2-12 \mathrm{pH}$. The HSPICE simulation results for the proposed circuit show well working and appropriate to be used as an ISFET $\mathrm{pH}$ sensor readout circuit.

\section{REFERENCES}

[1] P. Bergveld, "ISFET, Theory and Practice," in Proc. IEEE Sensor Conference, Toronto, pp. 1-26, 2003.

[2] A. Morgenshtein, L. S. Boreysha, U. Dinnar, C. G. Jakobson, and Y. Nemirovsky, "Wheatstone-Bridge readout interface for ISFET/REFET applications," Sensors and Actuators B: Chemical, vol. 98, no. 1, pp. 18-27, 2004

[3] B. Palan, F.V. Santos, J. M. Karam, B. Courtois, and M. Husak, "New ISFET sensor interface circuit for biomedical applications," Sensors and Actuators B: Chemical, vol. 57, no.3, pp. 63-68, 1999.

[4] L. Shepherd, P. Georgiou, and C. Toumazou, "A novel voltage-clamped CMOS ISFET sensor interface," in Proc. of IEEE International Symposium on Circuits and Systems ( ISCAS), pp. 3331-3334, 2007.

[5] S. Martinoia and W. Chunhua, and Z. Yan, "A Novel Current-Mode Readout Circuit for ISFET Sensor," IEEE Asia Pacific Conference on Circuits and Systems, APCCAS, pp. 407-410, 2008.

[6] Y. H. Ghallab, W. Badawy, and Karan V. I. S. Kaler, "A novel PH sensor using differential ISFET current mode read-out circuit," in Proceedings of the International Conference on MEMS, NANO and Smart Systems, pp. 255-258, 2003.

[7] Y. Liu and C. Toumazou, "An ISFET based sensing array with sensor offset compensation and $\mathrm{pH}$ sensitivity enhancement," in Proc. of IEEE International Symposium on Circuits and Systems (ISCAS), pp. 2283-2286, 2010.

[8] G. Massobrio, "A behavioral macromodel of the ISFET in SPICE," Sensors and Actuators B: Chemical, vol. 62, no. 3, pp. 182-189, 2000. 